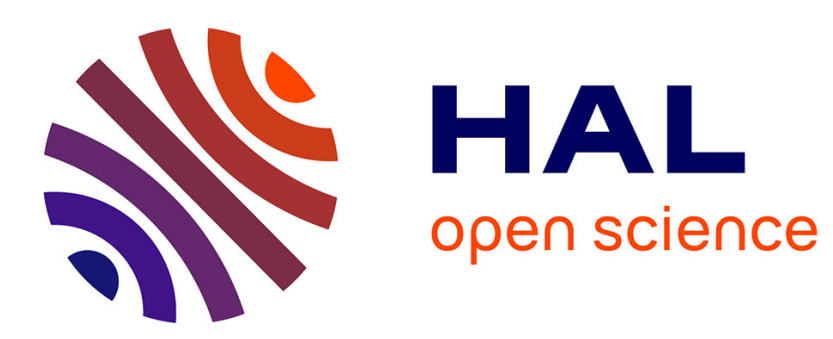

\title{
Model-based segmentation of the left main coronary bifurcation from 2D angiograms
}

\author{
Romain Lacroix, Raoul Florent, Vincent Auvray
}

\section{To cite this version:}

Romain Lacroix, Raoul Florent, Vincent Auvray. Model-based segmentation of the left main coronary bifurcation from 2D angiograms. Biomedical Imaging: Nano to Macro, 2012. 9th IEEE International Symposium on (ISBI 2012), May 2012, Barcelone, Spain. pp.780-783, 10.1109/ISBI.2012.6235664 . hal-00722949

\section{HAL Id: hal-00722949 \\ https://hal.science/hal-00722949}

Submitted on 6 Aug 2012

HAL is a multi-disciplinary open access archive for the deposit and dissemination of scientific research documents, whether they are published or not. The documents may come from teaching and research institutions in France or abroad, or from public or private research centers.
L'archive ouverte pluridisciplinaire HAL, est destinée au dépôt et à la diffusion de documents scientifiques de niveau recherche, publiés ou non, émanant des établissements d'enseignement et de recherche français ou étrangers, des laboratoires publics ou privés. 


\title{
MODEL-BASED SEGMENTATION OF THE LEFT MAIN CORONARY BIFURCATION FROM 2D ANGIOGRAMS
}

\author{
Romain Lacroix, Raoul Florent and Vincent Auvray \\ Medisys Research Lab, Philips Healthcare, 33 rue de Verdun, 92156 Suresnes, France
}

\begin{abstract}
This paper deals with the identification of the left main coronary bifurcation (LMB) in $2 D$ angiograms. We resort to a generic $3 D$ model of the $\mathrm{LMB}$ that is projected in the proper angulation to retrieve the expected $2 D$ skeleton of the LMB in the considered image. We derive from it an efficient discriminating filter that allows detecting the LMB, while being robust to anatomical variations. We demonstrate the potential of the method over a large database of 120 angiographic sequences from 15 patients. We also propose a method extension that exploits not only the present angiography, but also the previous ones, as acquired in the course of the exam.
\end{abstract} tation.

Index Terms - Coronary arteries, model-based segmen-

\section{INTRODUCTION}

This paper deals with the segmentation of the left main coronary bifurcation (LMB) in $2 D$ angiograms, which is the intersaection of the left main artery, the circumflex artery and the left anterior descending artery (Fig.1a).

The explicit positioning of that anatomical point is needed in some applications, for instance to normalize perfusion measures [1]. It might also be useful as a starting point to segment the whole coronary tree. Indeed, one of the main reasons why tracking-based vessel segmentation methods are much less popular in $2 D$ than in $3 D$ [2] is that most applications in the former case need to be real-time, and thus require an automatic and robust initialization.

Detecting the LMB from 2D angiographies is a challenging

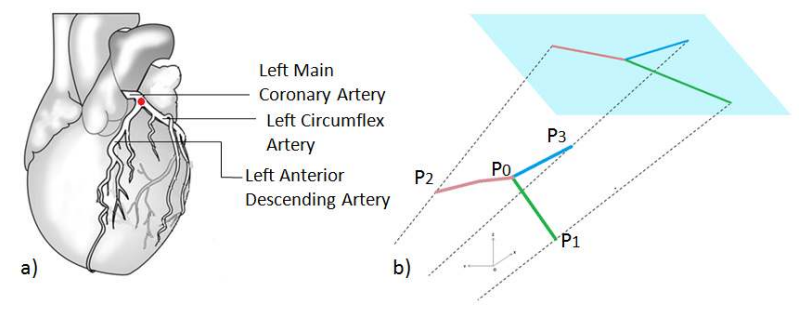

Fig. 1. a) Coronary arteries and left main bifurcation LMB (red point). b) 3D model of the LMB and its projection task. Typical angiographies present numerous contrasted objects (catheters, sternal wires, other vascular branches, etc) that can easily be mistaken for vessel bifurcations. Furthermore, the LMB has a variable shape in $2 D$, depending on the projection view (Fig.4).

In this paper, we resort to a generic (as opposed to patientspecific) model of the LMB, extracted from the coronary model proposed by Dodge [3]. From the projection of this $3 D$ model, we derive a $2 D$ filter that allows the fast and reliable identification of the LMB in the image.

This paper is organized as follows. Section 2 describes the $2 D$ filter derived from the $3 D$ LMB model, focusing on its ability to handle LMB's anatomical variability. The detection quality is studied over 15 patients and 120 sequences. Section 3 presents a method extension that not only relies on the current angiography, but also on the other ones as acquired the examination of the considered patient. Section 4 contains concluding remarks.

\section{MODEL-BASED SEGMENTATION OF THE LEFT MAIN CORONARY BIFURCATION FROM ONE 2D ANGIOGRAMS}

\subsection{Filtering strategy}

Dodge developed in [3] a geometrical model of the coronary artery tree by identifying points of interest on bi-planar X-ray images, yielding mean positions. In our case, we consider the four points defining the LMB: $\left(P_{0}\right)$ and the first segment of the three arteries originating from it $\left(P_{1}, P_{2}, P_{3}\right.$, Fig.1b).

Projecting that $3 D$ model under the current system angulation, we get a $2 D$ skeleton of the expected LMB centerline in the current angiography, from which we design a $2 D$ filter enhancing the LMB. Ridge filters [4] have proven well suited to enhance vessels. The ridge filter of the image $I$ in the direction $\theta$ and at the scale $\sigma$ at pixel $\mathbf{p}$, reads:

$$
R_{\sigma}^{\theta}(\mathbf{p})=L_{\theta} * G_{\sigma} * I(\mathbf{p})
$$

where $G_{\sigma}$ is the Gaussian filtering with a $\operatorname{kernel} \sigma$, and $L_{\theta}$ the second order derivative in the direction $\theta$.

To detect the LMB, we compute the response of the 3 expected branches to a ridge filter, oriented in the direction given by the model. A linear combination of those 3 filters is not 
discriminative enough since vessels responding to only 1 or 2 of the filters could dominate bifurcations. We therefore resort to the minimum response of the 3 branches as the composite filter:

$$
F_{L M B}^{1}(\mathbf{p})=\min _{i \in[1,3]}\left(R_{\sigma}^{\theta_{i}}\left(\mathbf{p}+\alpha \overrightarrow{P_{0} P_{i}}\right)\right)
$$

with $\theta_{i}$ the orientation of the vector $\overrightarrow{P_{0} P_{i}}$, and $\alpha$ a parameter setting the kernel positions on each branch.

Actually, we obtained better results by considering a series of aligned ridge filters for each branch, which is equivalent to 'elongating' the ridge kernel:

$$
F_{L M B}^{2}(\mathbf{p})=\min _{i \in[1,3]}\left(\sum_{j \in[1,3]}\left(R_{\sigma}^{\theta_{i}}\left(\mathbf{p}+\alpha_{j} \overrightarrow{P_{0} P_{i}}\right)\right)\right)
$$

\subsection{Proposed filter}

In practice, we observe that, due to anatomical variability, the LMB does not always accurately match the model skeleton, and thus can respond poorly to $F_{L M B}^{2}$. We therefore take into account the uncertainty to the exact vessel angles in the final filter formulation (see Fig.2):

$$
\begin{aligned}
F_{L M B}(\mathbf{p})= & \min _{i \in[1,3]}\left(\sum _ { j \in [ 1 , 3 ] } \left(\max _{k \in\left[-n_{k}, n_{k}\right]}\right.\right. \\
& \left.\left(R_{\sigma}^{\theta_{i}}\left(\mathbf{p}+\alpha_{j} \overrightarrow{P_{0} P_{i}}+k \beta_{j}{\overrightarrow{P_{0} P_{i}}}^{*}\right)\right)\right)
\end{aligned}
$$

with ${\overrightarrow{P_{0} P_{i}}}^{*}$ the vector orthogonal to $\overrightarrow{P_{0} P_{i}}$. This way, we explore variations around the expected vessel angle by positioning a series of ridge kernels parallely to the mean expected position (Fig.2). The further away from the bifurcation, the larger the possible variations of the vessel, and the more kernel we introduce. This filter handles variability that is mostly

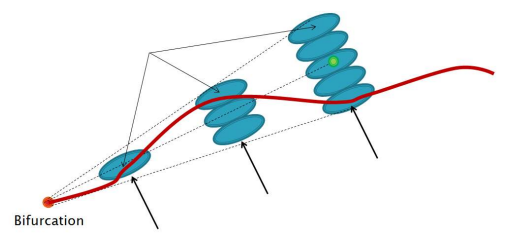

Fig. 2. Proposed filter (one branch only is plotted). The ellipsoids figure ridge kernels (Eq.4).

of anatomical nature. It is also robust to variations due to the projection direction (the $2 D$ LMB skeleton is not exactly the same, depending on the position in space of the $3 D$ model), and changes due to cardiac phases (Dodge's model is formally proposed for end diastole only).

Filter $F_{L M B}$ can be efficiently computed. For each branch, the ridge-filtered image is needed in one orientation only. Therefore, computing $F_{L M B}$ boils down to filtering the original image with 3 oriented ridge filters, and to combining their values (27 samples per pixel), which is real-time compatible.

\begin{tabular}{|c|c|c|}
\hline 1st cand. & 1st-2nd cand. & 1st-3rd cand. \\
\hline $64 \%$ & $88 \%$ & $92 \%$ \\
\hline
\end{tabular}

Table 1. Detection rate of the 2D LMB filter

To detect the LMB, we simply select the maximum of $F_{L M B}$ 's response. Alternatively, we can select the $n$ candidates by keeping the $n$ maxima of $F_{L M B}$ after a nonmaximum suppression, and identify the best one later on in the algorithmic pipeline.

\subsection{Detection results}

The detection quality of $F_{L M B}$ was evaluated over 120 angiographies from 15 patients. Table 1 presents the detection rate in the cases where the best 1,2 and 3 responses of the LMB maps are selected. We also show some detection results on Fig.4.

Given the large variety of the addressed configurations (low contrast, disturbing devices, see top two rows of Fig.4), the reported detection rate with 3 candidates $(92 \%)$ is excellent. Furthermore, the generic method presented here does not include temporal regularization nor geometric priors on the LMB location, which would certainly improve detection.

However, the detection quality with 1 candidate is only of $64 \%$. It is mostly due to the fact that other portions of the vasculature can occasionally match the projected LMB skeletton, and thus respond strongly to $F_{L M B}$ as well (third row on Fig.4). This calls for a policy where the best 3 candidates are selected at this point, and the final selection is made later on in the algorithm pipeline (for instance, after three candidates coronary tree segmentations have been extracted).

The detection fails in a few cases with dominant disturbing devices, and when the real LMB does not exhibit the expected shape (Fig.4). Further clinical analyses are needed to better understand whether the latter is due to non-typical patient positioning on the table, or to large anatomical variability.

\section{MODEL-BASED SEGMENTATION OF THE LEFT MAIN CORONARY BIFURCATION FROM SEVERAL 2D ANGIOGRAMS}

\subsection{Join LMB identification}

We have explored a possible extension of the 2D $F_{L M B}$ filtering for an improved LMB identification, in which we propose to exploit the previously acquired angiographies. A typical cathlab examination indeed begins with a series of $5-10$ angiographies, during which the clinician observes the vasculature from various perspectives to perform an accurate diagnosis. If no motion occurs during this set of exams, the LMB in the different images should correspond to the same $3 D$ space position. We can then position it jointly in 
the different angiographies (Fig.3). This constrains more the problem and thus should allow to reach a higher detection rate (while being transparent to the cathlab workflow).

Now, breathing and cardiac motions might occur between

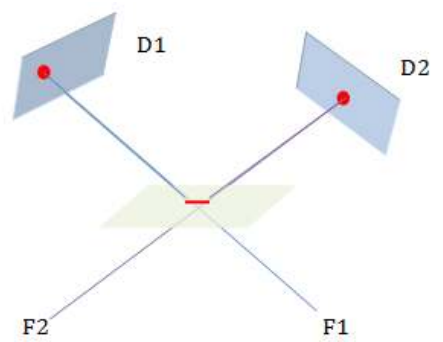

Fig. 3. LMB positioned coherently in different 2D images.

angiographies. Global table motions can be compensated for from the system data. In that case, the 3D geometrical constrain must be relaxed.

We select the $m$ best $2 D F_{L M B}$ responses on each of the $n$ considered angios. We consider each $n$-uplet of candidates, and assess whether they can anatomically originate from the same physical point. Basically, we retrace the different source-detector pixel rays, and assess whether they are sufficiently close together to account for breathing.

Each acceptable n-uplet is then associated a composite score based on the $2 D F_{L M B}$ scores. The anatomically acceptable combination having the highest score is finally detected - on each angiography.

\subsection{Results}

Unfortunately, the table motion was not part of our database. To test our multi-angiography approach, we had to manually estimate the global translation of the heart between the angiogram acquisitions (by clicking on the LMB on each of them), to correct for it, and then to add a simulated breathing. We relied on [5] to simulate realistic breathing amplitude differences (Gaussian law $\sigma=6 \mathrm{~mm}$ in $\mathrm{X}, \sigma=10 \mathrm{~mm}$ in Y). The detection results are reported in Tab.2 where $n=2$ angiographies are considered $(m=10)$. The database is not exactly the same than in subsection 2.3 since the angiograms that could not be paired with other angiograms (with a minimal C-arm difference of $20^{\circ}$ ) were discarded. This explains why the detection scores with one angiogram only are not identical to Tab.1.

When no simulated breathing is added (ie, every motion is compensated for), the test boils down to simulating biplane acquisitions. We report then a moderate detection improvement of $7 \%$ (with one candidate pair). The 3D geometrical constraint does not limit the number of valid pairs as much as expected. We measured that $18 \%$ of the pairing were accepted (range $7 \%-28 \%$, depending on the $\mathrm{C}$-arm angulations).

When the breathing was simulated, the $3 \mathrm{D}$ condition was re-

\begin{tabular}{|c|c|c|c|}
\hline Configuration & 1st cand. & 1st-2nd cand. & 1st-3rd cand. \\
\hline \hline One angio & $67 \%$ & $85 \%$ & $92 \%$ \\
Biplane & $74 \%$ & $89 \%$ & $94 \%$ \\
Two angios & $70 \%$ & $86 \%$ & $93 \%$ \\
\hline
\end{tabular}

Table 2. Detection rate of the 2D LMB filter (108 sequences)

laxed and more pairings were accepted (average 58\%, range $29 \%-91 \%$ ). This explains the smaller detection improvement of $3 \%$ reported in Tab.2. Similar minor improvements were measured in the case of 3 angiograms.

\section{CONCLUSION}

To identify the LMB in $2 D$ angiograms, we resort to a generic $3 D$ model of the LMB that is projected in the proper angulation to estimate the expected $2 D$ skeleton of the LMB in the considered image. We have derived from it an efficient discriminating filter that allows to detect the LMB. An excellent detection rate of $92 \%$ is reported (over a database of 120 sequences) when the 3 best scores are selected.

We have proposed an extension of our method that exploits the previously acquired angiographies to further constrain the localization of the LMB. We report only modest detection improvements, but without entailing any further constraint on the clinician. We now plan to exploit the full generic model of the heart to jointly segment and label the complete coronary tree in $2 D$ angiographic images.

\section{REFERENCES}

[1] J.Liénard and R.Vaillant, "Quantitative tool for the assessment of myocardial perfusion during X-ray angiographic procedures," in FIMH'09, 2009, pp. 124-133.

[2] D.Lesage, E.Angelini, I.Bloch, and G.Funka-Lea, "Bayesian maximal paths for coronary artery segmentation from 3D CT angiograms," in MICCAI, 2009, vol. 1, pp. 222-229.

[3] JT.Dodge, BG.Brown, EL.Bolson, and HT.Dodge, "Intrathoracic spatial location of specified coronary segments on the normal human heart," Circulation, vol. 78, pp. 1167-1180, 1988.

[4] A.F. Frangi, W.J. Niessen, K.L. Vincken, and M.A. Viergever, "Multiscale vessel enhancement filtering," in LNCS(1496), MICCAI’98, 1998, pp. 130-138.

[5] R.Jagsi, J.M.Moran, M.L.Kessler, R.B.Marsh, J.M.Balter, and L.J.Pierce, "Respiratory motion of the heart and positional reproducibility under active breathing control," Int.J. Oncol. Biol.Phys., vol. 68, no. 1, pp. 253-258, may 2007. 

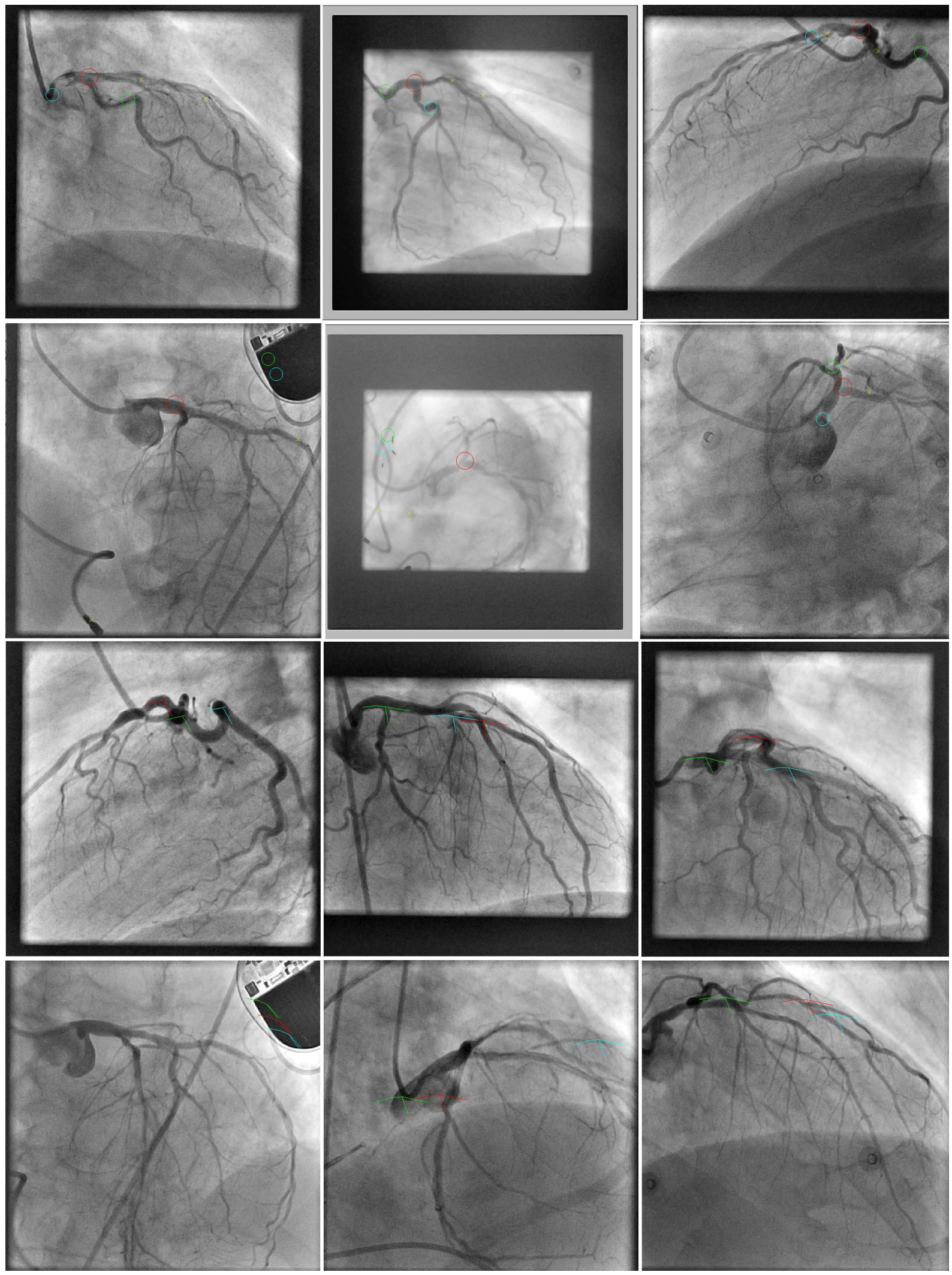

Fig. 4. Detection results with 2D filter $F_{L M B}$. In red the first maximum, green the second, blue the third. Top two rows: correct detection is the 1st maximum, including in difficult configurations (pacemaker, electrodes, low contrast, contrast backflow); third row: correct detection is the 2nd maximum, mostly because other parts of the vasculature match the tripod shape; fourth row: detection failure, either due to disturbing device, or because the real LMB does not exhibit the expected shape. The projected tripod is plotted on the last two rows to help interpretation. 